\title{
RESPUESTA HIDROLÓGICA DE UNA CUENCA FORESTAL EN LA MONTAÑA MEDIA PIRENAICA: EL CASO DE SAN SALVADOR
}

\author{
M.P. SERRANO MUELA, D. REGÜÉS, J. LATRON, \\ C. MARTÍ BONO, N. LANA-RENAULT y E. NADAL ROMERO \\ Instituto Pirenaico de Ecología, CSIC, Campus de Aula Dei, apartado 202, 50080- Zaragoza \\ Correo electrónico de contacto: pili@ipe.csic.es
}

\begin{abstract}
RESUMEN. Este trabajo expone las principales características hidrológicas de la cuenca experimental de San Salvador, un espacio representativo de la montaña media pirenaica muy poco afectada por la acción humana. La cuenca se caracteriza por presentar en la mayor parte de su superficie una densa masa boscosa que condiciona la respuesta hidrológica a cualquier evento pluviométrico. La cuenca de San Salvador fue monitorizada en 1999 mediante la instalación de una estación de aforo y una estación meteorológica. Entre septiembre de 1999 y junio de 2005 se han seleccionado 40 crecidas que se han utilizado para analizar las principales pautas de la respuesta hidrológica de la cuenca y jerarquizar los factores que mejor explican esa respuesta. Los resultados demuestran diferencias con respecto a ambientes mucho más afectados por modificaciones antrópicas, especialmente el gran peso de las condiciones de humedad antecedente. La intensidad y el volumen de precipitación tienen un papel poco relevante para explicar las características de la respuesta hidrológica.
\end{abstract}

ABSTRACT. This paper studies the main hydrological characteristics of the San Salvador experimental catchment, a representative area in the middle mountain of the Pyrenees, scarcely affected by human activities. The basin is characterized by the presence of a dense forest cover that conditions the hydrological response against any rainstorm event. The San Salvador catchment has been monitored since 1999 by means of a gauging station and a weather station, to quantify the runoff and sediment yield. 40 floods have been considered for the study, between September, 1999 and June, 2005. They have been used to analyse the principal hydrological patterns of the catchment and to rank the factors that better explain the hydrological response. The results show the existence of differences in comparison with environments much more affected by human disturbances, especially due to the importance of antecedent wetness conditions. The intensity and volume of rainfalls have a no relevant role to explain the characteristics of the hydrological response. 
Palabras clave: Hidrología ambiental, cuenca experimental, relaciones precipitaciónescorrentía, hidrograma de crecida, Pirineo central español.

Key words: Environmental hydrology, experimental catchment, rainfall-runoff relationships, flood hydrograph, Spanish Central Pyrenees.

Enviado el 10 de septiembre de 2005

Aceptado el 15 de noviembre de 2005

\section{Introducción}

Los espacios forestales constituyen, particularmente dentro del ámbito mediterráneo, ambientes clave para el sostenimiento del equilibrio hidrológico, más aún en un escenario de cambio climático que puede afectar directa o indirectamente a la generación de escorrentía (Cosandey et al., 2004).

Es bien conocido que los recursos hídricos no son independientes de las condiciones ambientales. Las características climáticas (volumen, intensidad y distribución de las precipitaciones), la litología, las pendientes y la cubierta vegetal explican gran parte de las diferencias de régimen hidrológico entre cuencas. Hoy se acepta de manera general que la vegetación y los usos del suelo tienen una gran responsabilidad en la cantidad y la calidad de los recursos hídricos, y que los cambios que introduce el hombre provocan alteraciones muy rápidas en el régimen fluvial (Beguería Portugués et al., 2003; Brown et al., 2005). No obstante, el papel concreto del bosque en el ciclo hidrológico ha sido y sigue siendo un tema muy controvertido (Hibbert, 1967; Cosandey et al., 2004; Andréassian, 2004). Ello obliga a estudiar de forma muy detallada y espacialmente distribuida, el funcionamiento hidrológico bajo diferentes cubiertas, especialmente el papel de la interceptación, la infiltración, el almacenamiento de agua en el suelo y su agotamiento, y su influencia en la respuesta hidrológica durante eventos hidrológicos.

Los estudios hidrológicos se basan frecuentemente en cuencas experimentales donde se controlan las entradas (en forma de precipitaciones) y las salidas (en forma de caudal de evapotranspiración y de sedimento). Las cuencas permiten valorar los efectos de distintas cubiertas vegetales (Hibbert, 1967; Bosch \& Hewlett, 1982; García Ruiz \& Gallart, 1997) y las relaciones entre las entradas y las salidas. Esa información sirve para el desarrollo y la evaluación de modelos hidrológicos que puedan predecir la evolución futura de las salidas en un escenario de Cambio Global.

Este trabajo tiene por objeto estudiar el funcionamiento hidrológico de la cuenca forestal de San Salvador, definir la respuesta hidrológica frente a eventos pluviométricos de diferente intensidad y determinar los factores que mejor explican el tipo de respuesta. El interés del estudio es doble: (i) debe aportar información sobre el funcionamiento hidrológico de un área de montaña donde la cubierta vegetal no ha sido eliminada y (ii) debe aportar datos sobre la posible tendencia futura de muchas cuencas en la montaña media pirenaica, dada la más que probable expansión de la cubierta forestal debido al abandono de la explotación agrícola en laderas. 


\section{2. Área de estudio}

El barranco de San Salvador es afluente por la margen izquierda del río Estarrún, en el valle de Aísa (Fig.1)., y se encuentra en un ambiente forestal que contrasta con otros próximos, intensamente explotados en el pasado. Se trata de una cuenca con escasos contrastes topográficos entre umbría y solana, y con un fuerte gradiente altitudinal, de casi 500 metros entre la divisoria $(1270 \mathrm{~m})$ y la estación de aforo $(880 \mathrm{~m})$.

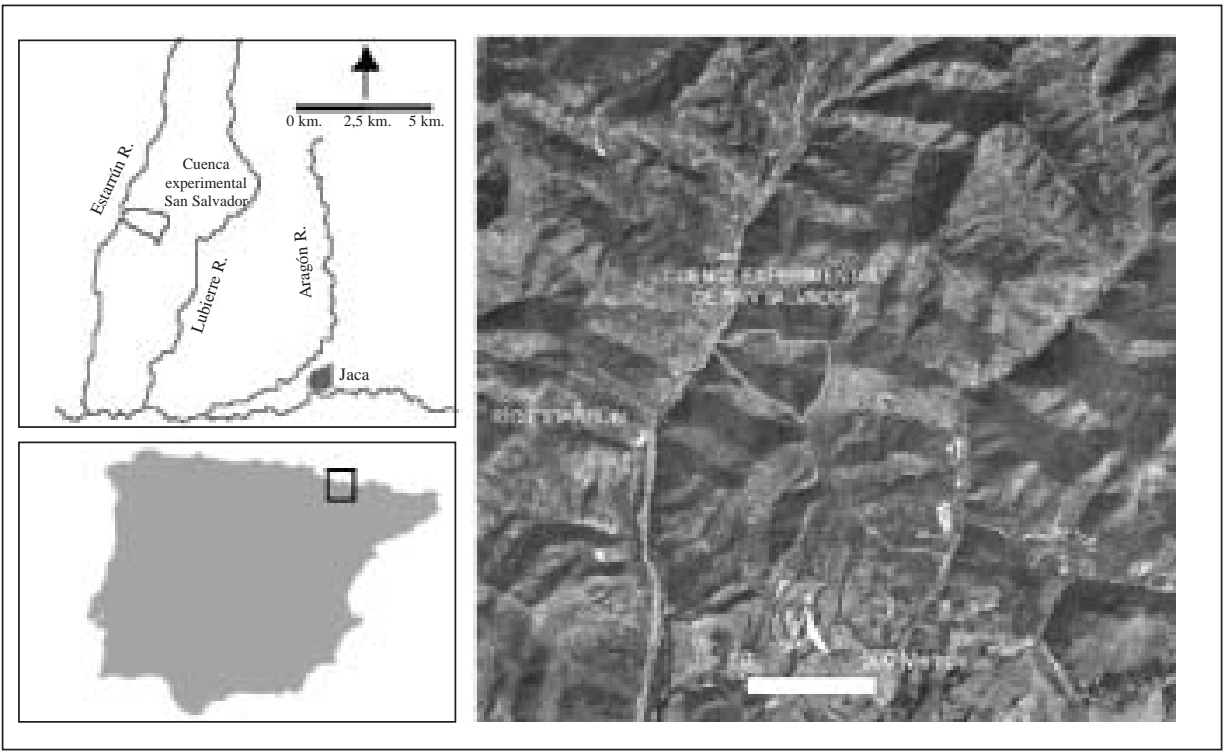

Figura 1. Localización de la Cuenca Experimental de San Salvador.

La cuenca, de 92 ha, se localiza en el Sector del Flysch Eoceno surpirenaico, lo que explica algunos de los rasgos topográficos y geomorfológicos más relevantes: (i) las vertientes muestran claros signos de regularización, hecho relacionado con la homogeneidad y relativa fragilidad del roquedo; (ii) las laderas aparecen recubiertas por un coluvión poco potente; (iii) la evolución más común de las vertientes se produce por medio de movimientos en masa superficiales que evolucionan hacia flujos o coladas de derrubios (García Ruiz \& Puigdefabregas, 1982); (iv) los materiales del flysch (areniscas y margas alternantes en bancos muy delgados) son ricos en material carbonatado que favorecen la formación de travertinos y microgours a lo largo del cauce.

El clima ha sido definido como "de montaña media mediterránea, con influencia atlántica de transición a submediterráneo" (Creus y Gil, 2001). La temperatura media anual oscila entre 9 y $10^{\circ} \mathrm{C}$, con una considerable amplitud térmica entre verano e 
invierno. Las temperaturas estivales son relativamente suaves. En invierno son frecuentes las heladas que se prolongan habitualmente desde noviembre hasta abril, aunque pueden producirse incluso a finales de mayo. La precipitación media anual registrada en la estación de Aísa, a una distancia aproximada de 6 kilómetros y localizada a 1100 m de altitud, es de $1212 \mathrm{~mm}$. El régimen anual muestra 2 periodos con importantes lluvias, uno en primavera y otro a mediados y finales de otoño. Existe un marcado descenso pluviométrico estival interrumpido ocasionalmente por tormentas intensas pero de corta duración. El invierno no es muy húmedo debido a la persistencia de situaciones anticiclónicas, aunque el paso de frentes por el norte de la Península Ibérica propicia la ocurrencia de abundantes nevadas, sobre todo en la parte alta del valle de Aísa. La acumulación de nieve es importante a partir de 1650-1700 m, coincidiendo con la posición de la isoterma $0^{\circ} \mathrm{C}$ durante la estación fría (García Ruiz et al., 1985; Del Barrio et al., 1990), por lo que en la cuenca de San Salvador no es frecuente la precipitación en forma de nieve.

La acusada influencia mediterránea se deja sentir en la vegetación de la cuenca de San Salvador. Su distribución está fuertemente marcada por la exposición. En umbría se distinguen dos ambientes diferentes. Por un lado, un pinar musgoso de pino silvestre (Pinus sylvestris) muy denso, con sotobosque compuesto mayoritariamente por boj (Buxus sempervirens). Por otro lado, destacan sectores poblados con hayas (Fagus sylvatica) que aparecen en los lugares más húmedos, normalmente en pequeñas concavidades topográficas que favorecen la formación de pequeños bosquetes galería. El sotobosque propio del hayedo es más pobre que el del pinar y lo componen especies como el acebo (Ilex aquifolium). De forma dispersa aparecen otras especies caducifolias, como arces (Acer opalus), avellanos (Corylus avellano) o tilos (Tylia platyphyllos). El manto de herbáceas es bastante denso, con especies acompañantes típicas de estos hábitats más húmedos (Hepatica nobilis, Fragaria vesca, Heleborus sp, Primula sp, etc.) Destaca igualmente la importante capa de musgos que acolcha el suelo, así como el potente manto de hojarasca acumulado.

En la exposición solana la vegetación se presenta menos densa en general, existiendo algunos claros heredados de la práctica de actividades agrícolas y ganaderas. La vegetación característica en esta ladera es el quejigal submediterráneo oriental o quejigal con boj (Monserrat, 1971), especie abundante de estos bosques. Junto con estas especies es frecuente hallar arces (Acer opalus), que prosperan con facilidad en estas áreas de solana. Además pueden encontrarse ejemplares dispersos de pino laricio de Austria (Pinus nigra subesp. nigra) en la parte más baja de la ladera, una especie que seguramente aquí fue introducida por el hombre ya que fue muy utilizada para repoblaciones masivas. El sotobosque de la solana lo forma fundamentalmente el boj, junto a especies de tipo espinoso y de porte bajo, como la aliaga (Genista scorpius), el enebro (Juniperus communis) y el erizón (Echinospartum horridum), éste último donde los suelos son mas someros, mezclándose con rosáceas, zarzas y majuelos (Crataegus monogyna). Las escasas superficies donde hubo campos de cultivo han sido recolonizadas casi totalmente por estas especies de matorral que poco a poco tienden a regenerar en quejigo. 
Los suelos descritos por Seeger et al. (2004) en la cuenca de Arnás (286 ha), muy próxima a la de San Salvador, muestran diferencias entre solana y umbría. Los suelos de solana están mucho menos desarrollados que en umbría, siendo en ésta el más representativo el kastanozem, caracterizado por ser bastante profundo y estar bien estructurado. En solana se encuentran desde regosoles superficiales a cambisoles más desarrollados. Todos ellos son muy ricos en material carbonatado.

\section{Equipamiento y métodos}

La cuenca de San Salvador fue monitorizada en el año 1999, aunque su funcionamiento fue inicialmente muy irregular. A partir del año 2001 se dispone de una serie de datos más continua. La instrumentación instalada en la cuenca permite disponer de información acerca de las entradas (precipitaciones) y salidas (caudal, evapotranspiración y sedimento), y es la siguiente (Fig. 2):

- 3 pluviómetros de balancín localizados a diferentes cotas para comprobar si el gradiente de altitud influye en la cantidad de lluvia y en su distribución temporal.

- Una estación de aforo donde un sensor de ultrasonidos mide la altura de agua (modelo Lundhal DCU-7110). La estación cuenta además con una trampa de sedimen-

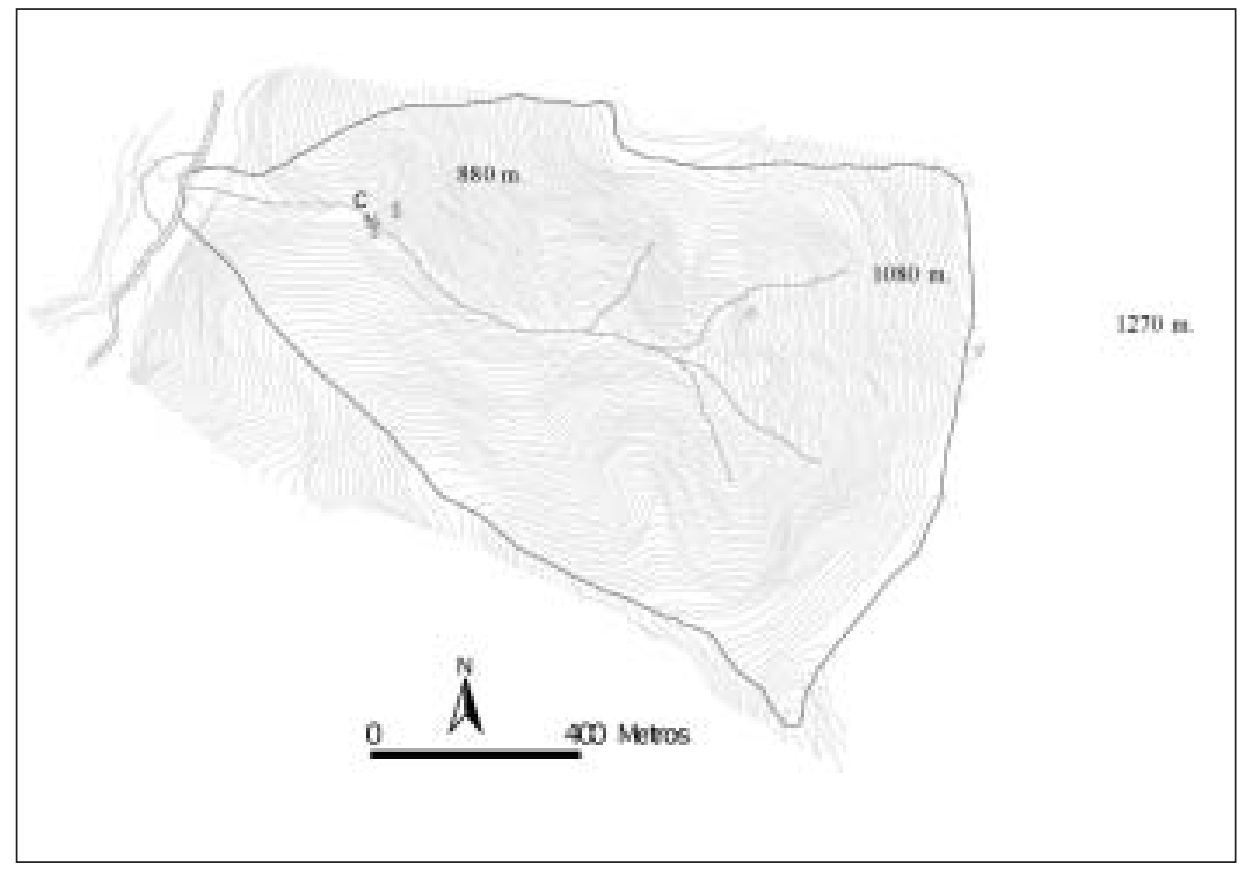

Figura 2. Localización del equipamiento de la cuenca de San Salvador. 
to, excavada en el cauce y forrada de aluminio, donde cae el sedimento que circula por el fondo del cauce en forma de carga de fondo (bedload) (con una capacidad aproximada para $700 \mathrm{Kg}$.). Un tomamuestras automático (modelo ISCO 3700), permite controlar el sedimento en suspensión y solución en el momento de la crecida. Tiene capacidad para 24 botellas de 1 litro cada una. Durante varios años la estación contó con un turbidímetro (LYX 800 PT1) para la medición de la turbidez del agua. Sin embargo, debido a la permanente deposición de carbonatos en el sensor óptico se optó por su desinstalación en 2003

- Una estación meteorológica situada junto al aforo de la cuenca. Es de tipo Campbell y está equipada con termómetro, higrómetro, anemómetro, piranómetro y pluviómetro.

- 2 Piezómetros (modelo Keller) para la medición de la profundidad del nivel freático.

Todos los instrumentos están conectados a colectores de datos que registran valores medios entre 5 y 20 minutos dependiendo de las variables.

$$
* * *
$$

Para este estudio se ha seleccionado un total de 40 crecidas, entre octubre de 1999 y septiembre de 2005. Algunos autores (Hewlett, 1982; Beven, 1991) aseguran que el estudio del hidrograma no puede dar por sí solo información acerca de la escorrentía producida y de los procesos que intervienen en su génesis. Hay que tener en cuenta que la elección de una metodología para selección de crecidas suele basarse en criterios un tanto arbitrarios. Es por ello que separar en el hidrograma la fracción que corresponde a la escorrentía superficial de la que pertenece a la escorrentía subterránea es una indeterminación. Para el presente estudio las crecidas se han identificado como aumentos en el caudal que superan en 1,5 veces el caudal de base anterior al inicio de la tormenta. El punto de comienzo corresponde a un cambio positivo y generalmente súbito de caudal, mientras que al final se fija en el momento en que el tramo descendente de la crecida cambia claramente de pendiente o se estabiliza (García Ruiz et al., 2005).

Una vez individualizadas las 40 crecidas, se definieron las variables derivadas de cada una de las crecidas, incluyendo información pluviométrica y de caudal. Tales variables son las siguientes:

- Precipitación total (PTOT).

- Tiempo de respuesta (TRESPUESTA)

- Intensidad media de precipitación (IPMED).

- Intensidad máxima de precipitación en 5 minutos (IPMAX_5).

- Caudal medio durante la crecida (QMED).

- Pico de caudal (QMAX_5).

- Coeficiente de escorrentía (COEFE).

- Caudal de base inicial (BF0). 
- Antecedentes de precipitación en 1 día (AP1D).

- Antecedentes de precipitación en 3 días (AP3D).

- Antecedentes de precipitación en 7 días (AP7D).

- Antecedentes de precipitación en 15 días (AP15D).

- Antecedentes de precipitación en 21 días (AP21D).

Se construyó así una base de datos que permitió establecer una matriz de correlación entre las variables. Posteriormente se utilizó una técnica multivariante (cluster) con el propósito de agrupar las crecidas seleccionadas para el periodo de estudio, basándose en las características de las mismas. Con los resultados obtenidos se realizó un análisis de varianza (ANOVA) para comprobar en qué medida era significativa esa agrupación. Finalmente se acudió a la técnica de análisis factorial de componentes principales para conocer y jerarquizar los factores que determinan la generación de crecidas.

\section{Resultados}

\subsection{Respuesta hidrológica a escala anual}

En el periodo estudiado existen años hidrológicos con diferencias muy acusadas, debido a la variabilidad de las precipitaciones. El año hidrológico más húmedo fue 2003-2004, en el que la precipitación llegó hasta casi 900 mm, mientras que el más seco ha sido el último, 2004-2005, en el que la precipitación registrada ha sido de $676 \mathrm{~mm}$. Este último valor de precipitación ha dado lugar a caudales muy bajos en el barranco de San Salvador, con un caudal de base alrededor de $21 \mathrm{~s}^{-1}$, y picos de crecida que prácticamente no han superado los $20 \mathrm{l} \mathrm{s}^{-1}$. Esta última cifra es casi equivalente al caudal medio habitual en años en que no hay sequía. De hecho, el caudal base medio del barranco está alrededor de 10 a $151 \mathrm{~s}^{-1}$, con picos de crecida que superan con frecuencia los $1001 \mathrm{~s}^{-1}$.

La Fig. 3 corrobora la marcada estacionalidad de las precipitaciones. Como regla general existen dos periodos húmedos, en otoño y primavera, quedando en ocasiones unidos en un mismo periodo, ya que la estación invernal no suele ser muy seca.

Las primeras lluvias al inicio del año hidrológico no producen respuesta, o es muy limitada incluso aunque la lluvia sea relativamente intensa. Esto se debe probablemente al hecho de que el suelo de la cuenca está seco tras el periodo estival, por la falta de precipitación y el consumo de agua por parte de la vegetación. Las lluvias generadas en noviembre son las primeras que producen respuesta de caudal, una vez que el suelo ha empezado a recargar sus reservas.

El funcionamiento hidrológico invernal es muy irregular, con años secos frente a otros más húmedos, dependiendo del régimen anual de precipitaciones. En la Fig. 3 puede apreciarse, por ejemplo, que el año 1999-2000 fue seco desde diciembre hasta abril, como también el año 2004-2005; el año 2003-2004 muestra un invierno algo húmedo y el año 2002-2003 puede considerarse muy húmedo en invierno. 


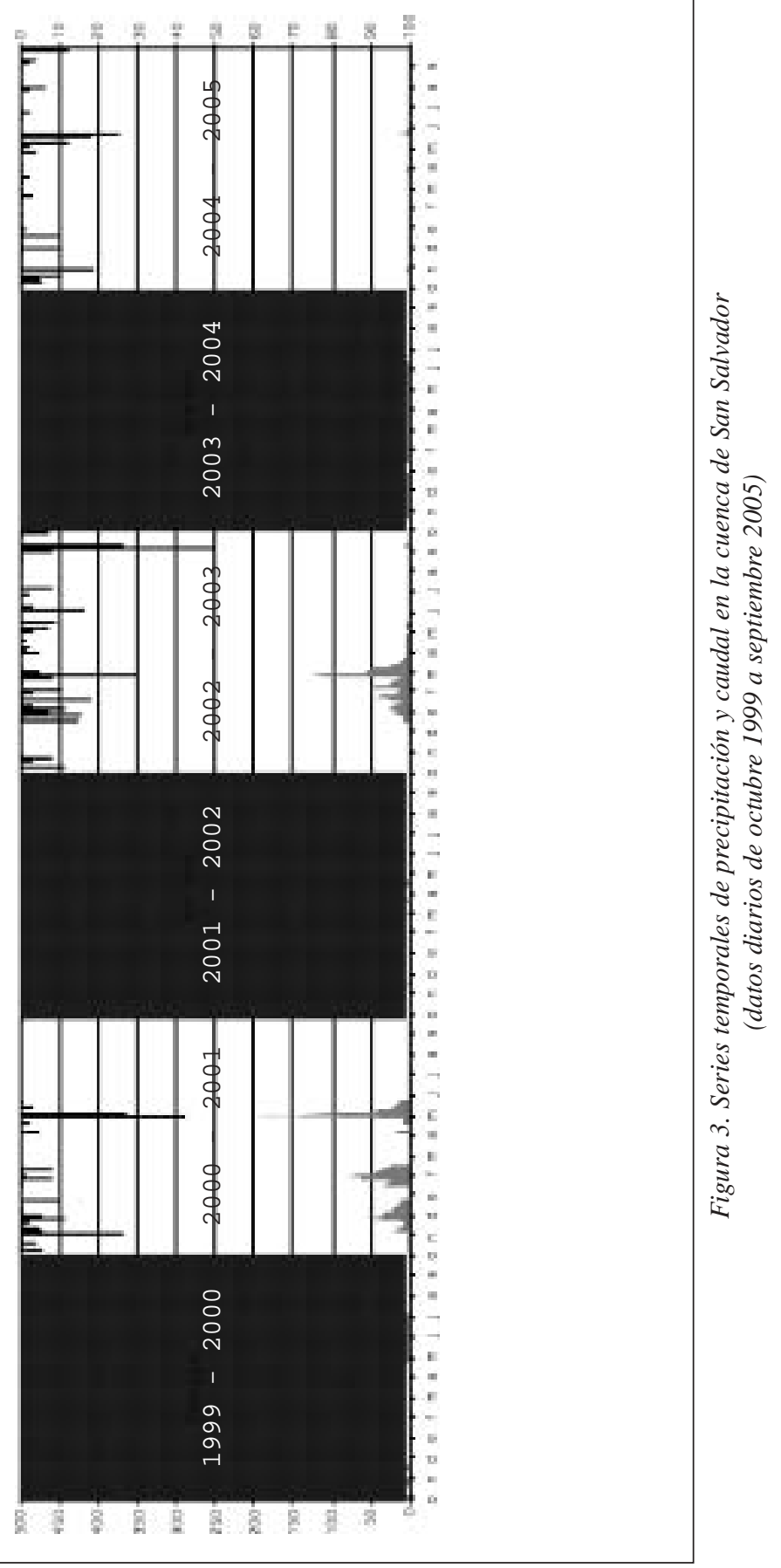




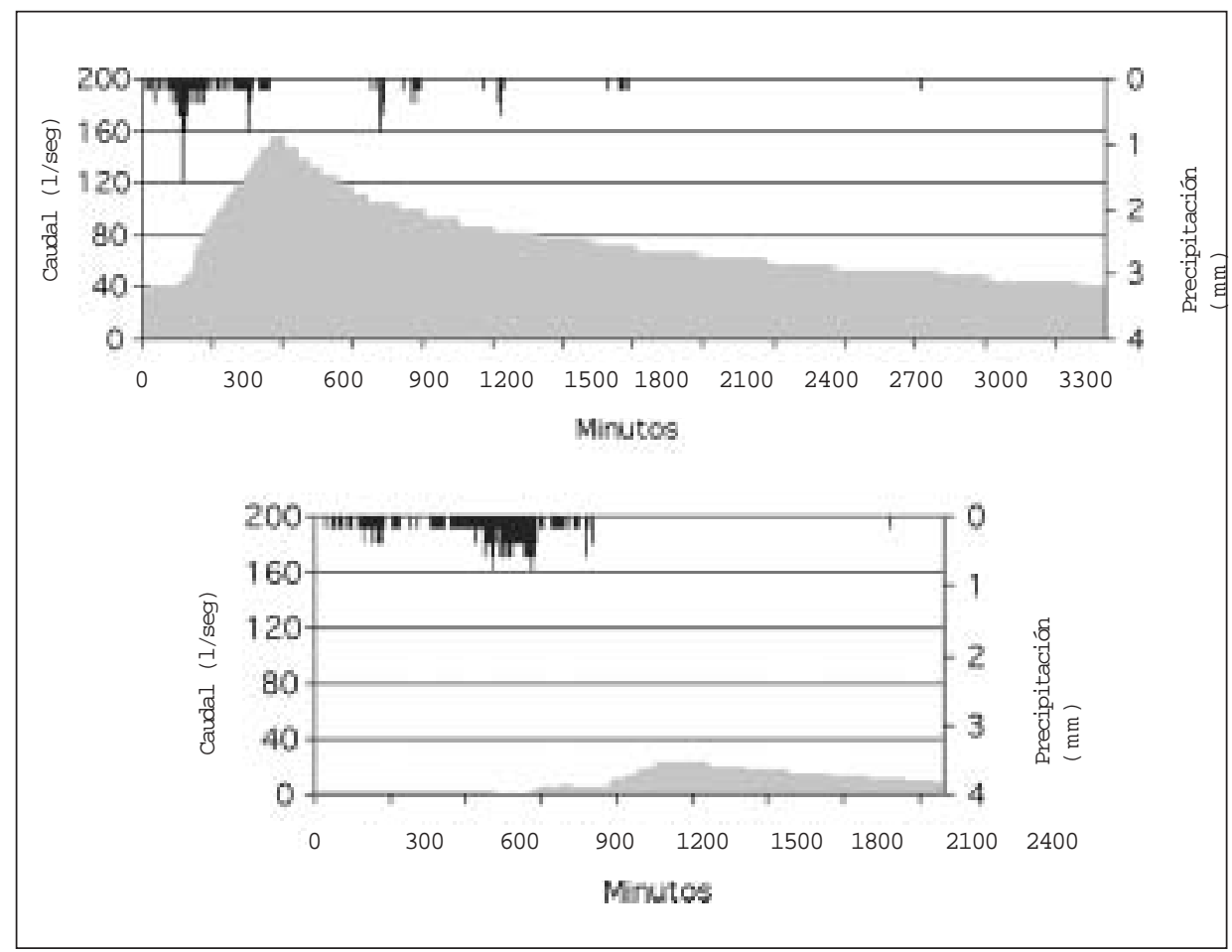

Figura 4. Respuesta de San Salvador ante condiciones de humedad antecedente diferente. 4a: 31 de octubre de 2003. 4b: 28 de febrero de 2002.

Por su parte, la primavera tiende a ser húmeda, aunque a veces puede ser relativamente seca, como en 2002-2003, o en 2004-2005 (que son sin embargo años con poca precipitación a lo largo de todo el año).

En verano, la respuesta hidrológica es casi inexistente o muy débil cuando concurren intensos eventos tormentosos con abundantes precipitaciones en los días anteriores.

La relación entre precipitación y escorrentía a lo largo del año está condicionada por la sucesión de eventos pluviométricos. Sin embargo, el estado antecedente de humedad del suelo juega posiblemente un papel relevante en el control de la respuesta hidrológica. En la Fig. 4 se manifiesta la diferente respuesta de la cuenca según sean las lluvias en días anteriores. En el primer ejemplo se observa una crecida generada por una lluvia de 13,6 mm en el contexto de estado hidrológico de la cuenca húmedo, con lluvias de $141 \mathrm{~mm}$ en los 21 días anteriores, lo que genera un acusado pico de caudal (278 $1 \mathrm{~s}^{-1}$ ) y una larga duración de la crecida. En el segundo caso, por el contrario, una precipitación de 38,2 mm sólo genera un pico de caudal de $221 \mathrm{~s}^{-1}$ debido a que las precipitaciones antecedentes son sólo de $20,8 \mathrm{~mm}$ en 21 días, además la crecida es de corta duración. 


\subsection{Respuesta hidrológica a escala de evento}

En primer lugar se quiso conocer la relación existente entre las variables de estudio. Los resultados más reveladores se muestran a continuación.

La relación entre la precipitación total del evento y el pico de caudal destaca por una baja correlación que carece de significación $\left(\mathrm{R}^{2}=0,047\right)$ (teniendo en cuenta que la correlación es significativa al nivel 0,01* y significante al nivel 0,05**). En la Fig. 5 se observa la elevada dispersión de los puntos, sin que pueda observarse una tendencia clara. Esto da una idea del complejo funcionamiento de la cuenca, ya que no se cumple lo que debería ser evidente: a medida que se incrementa la precipitación cabría esperar un mayor pico de caudal. Por el contrario, la misma figura muestra que para valores similares de precipitación la respuesta puede ser muy diferente. Por ejemplo, para una lluvia de $30 \mathrm{~mm}$ la respuesta hidrológica puede generar un pico de avenida casi inapreciable o llegar hasta $600 \mathrm{l} \mathrm{s}^{-1}$. Incluso con una lluvia de $20 \mathrm{~mm}$ puede no haber repuesta o acercarse a $12001 \mathrm{~s}^{-1}$. Esto quiere decir que la respuesta frente a una precipitación está condicionada por otros factores, además de la precipitación.

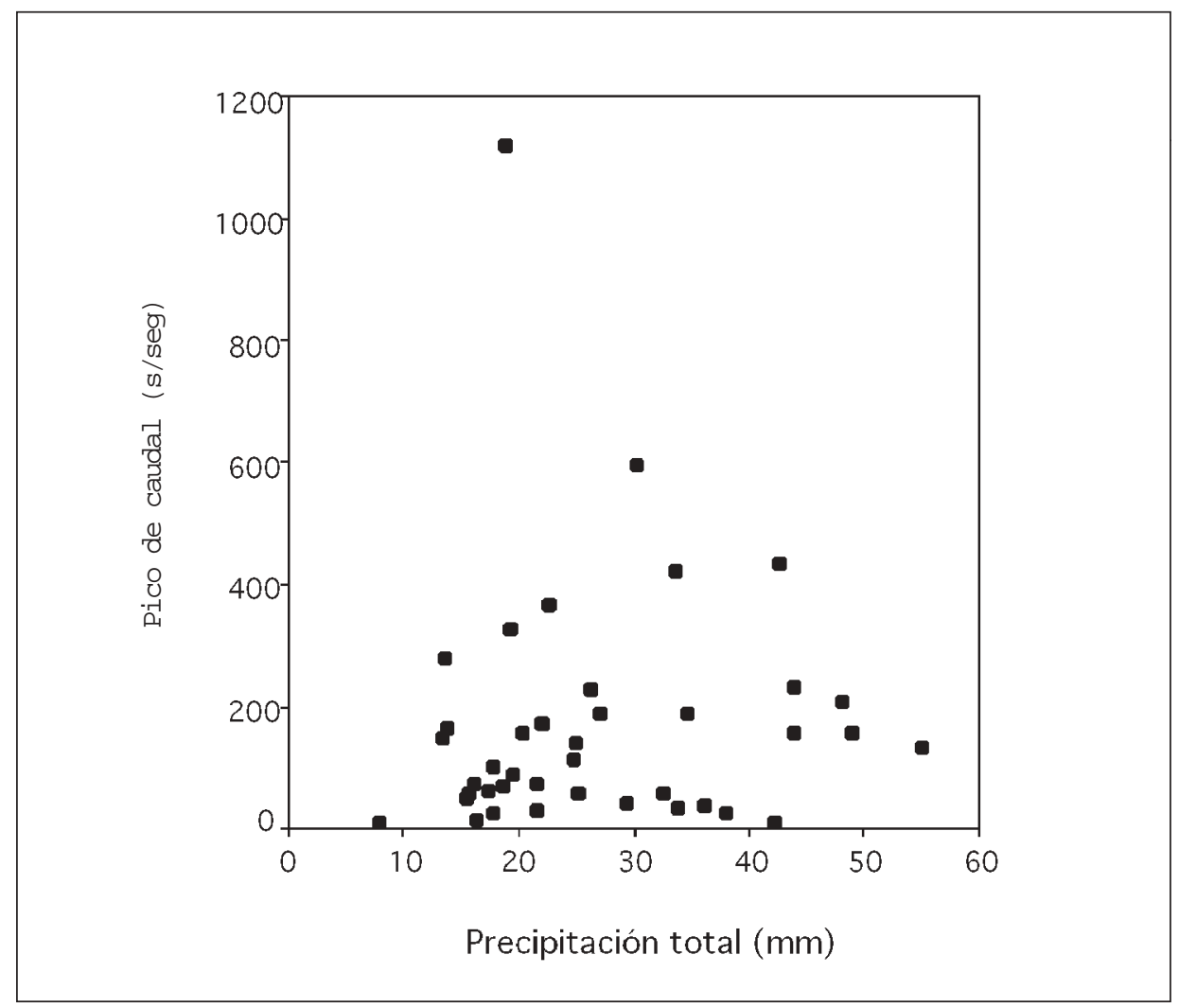

Figura 5. Relación entre precipitación y pico de caudal en las crecidas de la cuenca de San Salvador. 
El caudal de base desempeña un papel importante en la respuesta de la cuenca. El caudal al inicio de la crecida es un buen indicador de cómo reaccionará la cuenca ante cualquier evento de precipitación. Esta variable presenta muy buena correlación con el caudal medio y el pico de caudal en 5 minutos $\left(\mathrm{R}^{2}=0,75^{* *}\right.$ y $\mathrm{R}^{2}=0,78 * *$ respectivamente). La Fig. 6 refleja una tendencia muy positiva en la relación entre el caudal de base inicial y el caudal medio de la crecida. No obstante, en los casos más extremos sigue habiendo mucha dispersión. Por supuesto, el caudal de base inicial mantiene una buena relación con la lluvia registrada en los 15 días anteriores al comienzo de la avenida $\left(\mathrm{R}^{2}=0,676^{* *}\right.$ para 1 día, $\mathrm{R}^{2}=0,543 * *$ para 3 días, $\mathrm{R}^{2}=0,407 * *$ para 7 días y $\mathrm{R}^{2}=0,452 * *$ para 15 días anteriores). Este resultado, con una elevada significación, demuestra que la intensidad de la respuesta hidrológica depende en gran medida de las condiciones antecedentes, mientras que las características de la lluvia adquieren un papel secundario.

Si se separan las crecidas según la estación del año en que ocurren, todas las correlaciones adquieren valores más altos durante la estación húmeda. Lamentablemente, el número de casos en estación seca es de sólo 4, lo que impide cualquier relación estadística. En todo caso, el hecho de que las correlaciones mejoren si sólo se consideran las

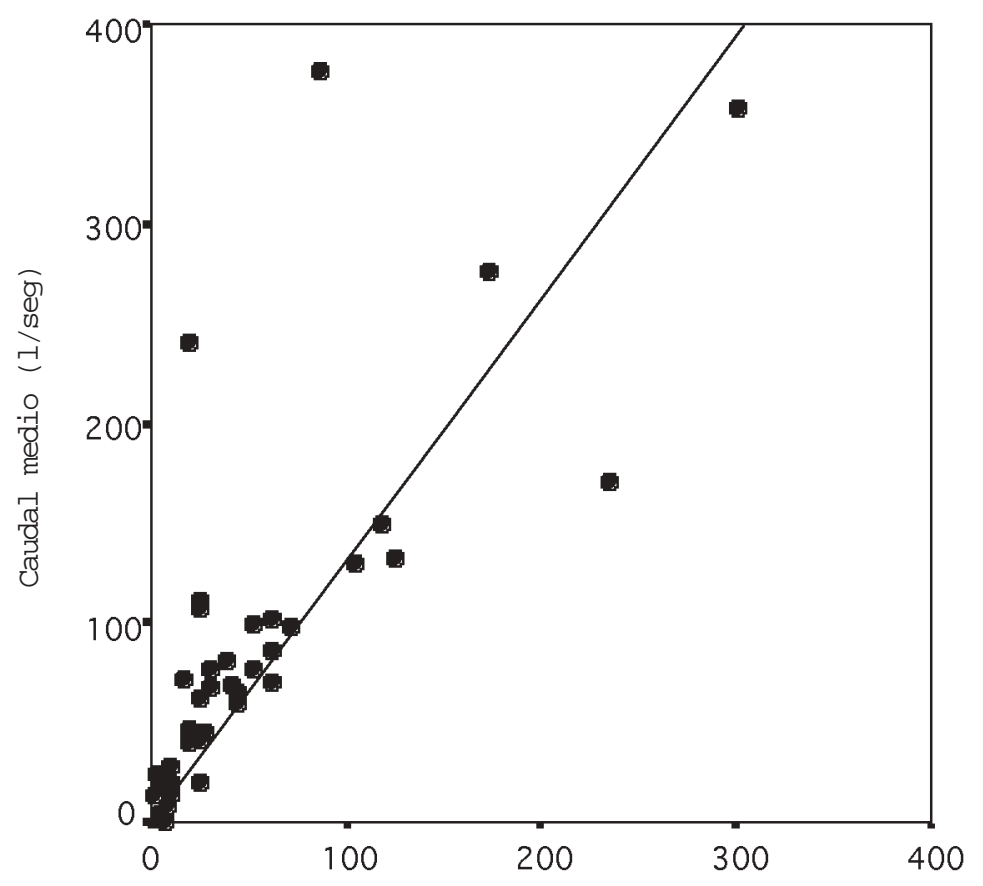

Fluio de base inicial

Figura 6. Relación entre caudal de base inicial y caudal medio en las crecidas de la cuenca de San Salvador. 
Tabla 1. Varianza de los 4 grupos de crecidas.

\begin{tabular}{|l|c|c|c|c|c|}
\hline & GRUPO 1 & GRUPO 2 & GRUPO 3 & GRUPO 4 & Sig. \\
\hline Precipitación total (mm) & 27,1 & 33,1 & 20,8 & 20,6 & 0,108 \\
Tiempo de respuesta (min) & 677 & 230 & 397 & 86 & 0 \\
Intensidad media pp (mm/h) & 57,8 & 144 & 68,5 & 128,1 & 0 \\
Intensidad máxima pp (mm/h) & 12 & 43 & 15 & 34 & 0,075 \\
Caudal medio durante la crecida (I/s) & 50 & 69 & 117 & 259 & 0 \\
Pico de caudal en 5 min (|/s) & 79 & 176 & 171 & 578 & 0 \\
Flujo de base inicial (|/s) & 24 & 27 & 80 & 187 & 0 \\
Antecedentes de precipitación en 1 día (mm) & 3,9 & 8,4 & 13,3 & 47,8 & 0 \\
Antecedentes de precipitación en 3 días (mm) & 13,2 & 17,9 & 54,9 & 54,4 & 0 \\
Antecedentes de precipitación en 7 días (mm) & 31,3 & 29,7 & 91,2 & 60,5 & 0 \\
Antecedentes de precipitación en 15 días (mm) & 55,2 & 46,5 & 124,3 & 97,1 & 0 \\
Antecedentes de precipitación en 21 días (mm) & 83,4 & 56,7 & 144,5 & 125,7 & 0 \\
\hline Número de casos & 19 & 10 & 7 & 4 & \\
\hline
\end{tabular}

crecidas de la estación húmeda, significa que las pocas crecidas de la estación seca contribuyen a empeorar esas relaciones. En el fondo eso quiere decir que la respuesta hidrológica en estación seca es muy aleatoria.

Una vez conocidas las variables que más relación presentan entre sí se efectuó un análisis cluster. Con esta técnica se ha pretendido diferenciar varios grupos de crecidas en función de las características de la precipitación y de los rasgos de la respuesta. El resultado obtenido proporciona cuatro grupos, con los que se realizó un análisis de varianza (ANOVA) que caracteriza los valores medios de las variables. Tal como se puede ver en la Tabla 1, todas las variables elegidas se muestran significativas, a excepción de los valores de intensidad (máxima en 5 minutos) y los de volumen de precipitación.

El Grupo 1 se define por unos valores medios de precipitación de $27 \mathrm{~mm}$ y una intensidad media y en 5 minutos muy baja. El tiempo de respuesta es el más largo de todos los grupos (11 horas), lo que está en parte condicionado por la baja intensidad (los valores más bajos de todos los grupos). Los valores medios de caudal medio y pico de caudal reflejan valores relativamente bajos (pico de caudal de 79,47 $1 \mathrm{~s}^{-1}$ ). La información del caudal de base inicial $\left(24 \mathrm{l} \mathrm{s}^{-1}\right)$ y las escasas lluvias antecedentes reflejan que la cuenca se encuentra en un estado bastante seco. Son crecidas producidas al inicio de las estaciones húmedas, por lo que gran parte de la lluvia es empleada en recuperar los acuíferos y sólo entonces se produce un incremento de caudal, que parece más relacionado con escorrentía subsuperficial y subterránea que superficial (Fig. 7a).

El Grupo 2 se caracteriza por los valores más altos de precipitación y de intensidad media y máxima en 5 minutos. Estas lluvias se producen cuando la cuenca no se encuentra muy húmeda. De hecho, las precipitaciones anteriores a la crecida no son muy abundantes. Por eso, y a pesar de que las precipitaciones son elevadas, la respuesta hidrológica es moderada, con pico de caudal de $175 \mathrm{l} \mathrm{s}^{-1}$ y un caudal medio relativamente bajo durante la crecida. Debido a la fuerte intensidad el tiempo de respuesta es relativamente rápido (4 horas). Son crecidas generadas por precipitaciones de poca duración y elevada intensidad (Fig. 7b), por lo que la respuesta suele ser rápida y corta. 


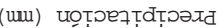

(unu) uọțeาțdțวəxd
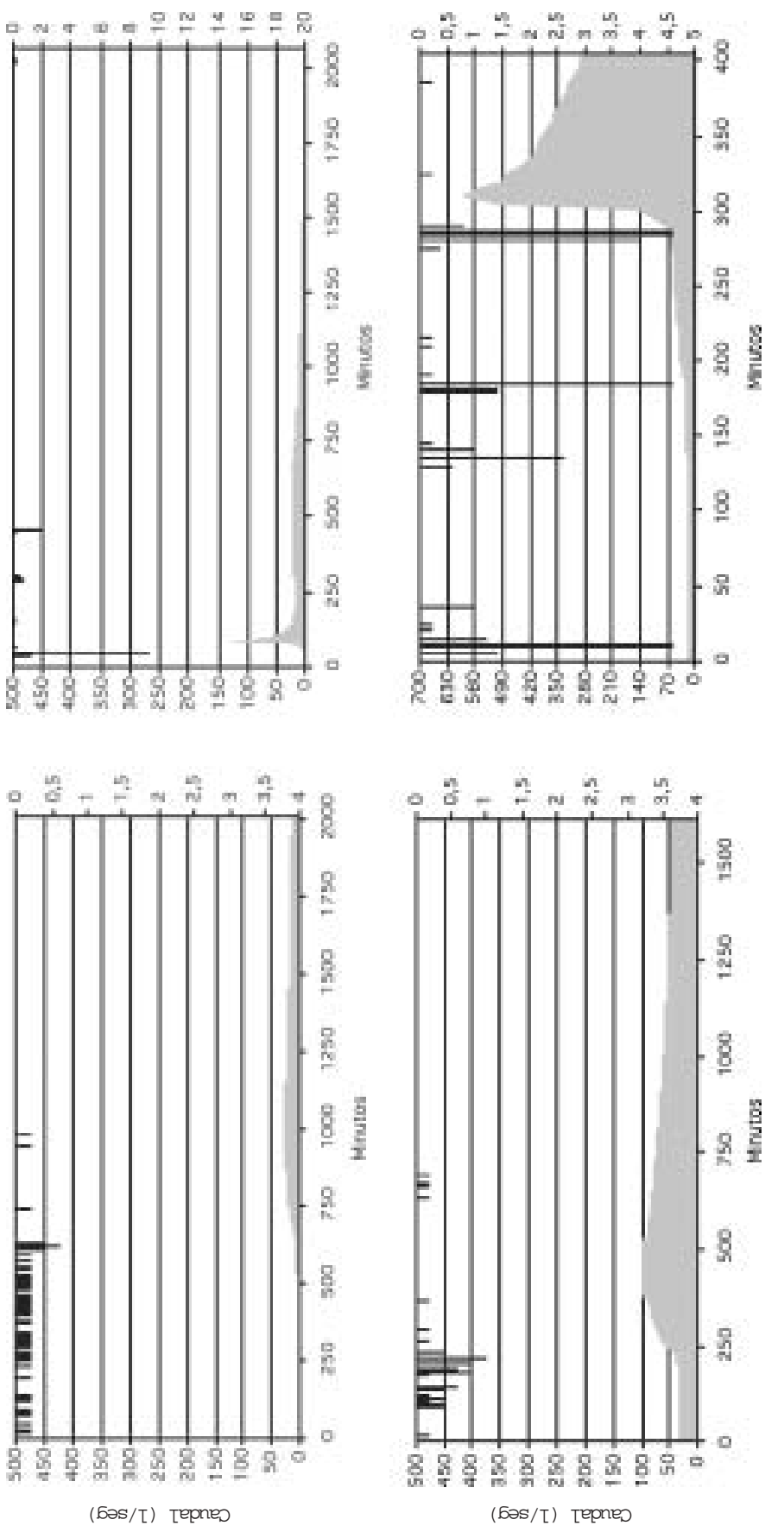

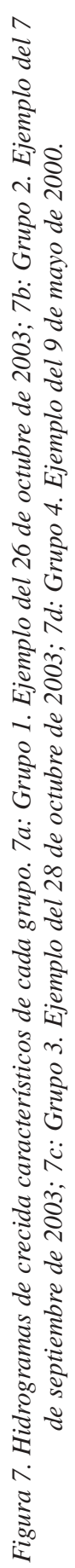


El Grupo 3 se caracteriza por valores de precipitación y de intensidad muy bajos. Sin embargo, el caudal de base al inicio de la crecida es elevado. Observando la lluvia antecedente se deduce que ha habido eventos importantes, sobre todo durante los 3 días antes del inicio de la crecida, e incluso hasta 3 semanas antes de la crecida. Esta precipitación antecedente es la que contribuye en mayor medida a la respuesta de la cuenca, que retrasa la salida de caudal debido a que la intensidad es menor, favoreciendo procesos de infiltración y la saturación del suelo. El tiempo de repuesta en este Grupo es de 6 horas. Son las crecidas producidas generalmente en las estaciones húmedas (Fig. 7c) y su respuesta tiende a ser moderada a condición de que la precipitación sea de baja intensidad y volumen. La figura del hidrograma es muy significativa: muestra un ligero incremento de caudal y luego éste se mantiene estable con ligera y prolongada tendencia decreciente.

El Grupo 4 se caracteriza por una rápida respuesta (menos de 1 hora y media, la más corta de todas) a pesar de que los valores de precipitación son los más bajos (20,6 $\mathrm{mm})$ similares al Grupo anterior. La intensidad media es alta $\left(128 \mathrm{~mm} \mathrm{~h}^{-1}\right)$. El caudal de base al inicio de la crecida es muy alto $\left(187 \mathrm{~s}^{-1}\right)$ respondiendo a una elevada precipitación antecedente, sobre todo durante los 3 días antes de inicio de la crecida. La consecuencia es un pico de crecida muy elevado, llegando en promedio a $5781 \mathrm{~s}^{-1}$, es decir, 3 veces más que cualquiera de los restantes tipos de crecidas. En definitiva, el estado de humedad de la cuenca, con abundantes lluvias anteriores, favorece una respuesta rápida y un pico de caudal muy acusado. Son las crecidas producidas al final de las estaciones húmedas (Fig. 7d), con lo que el hidrograma muestra un pico muy marcado y ascenso rápido, con un descenso también rápido inmediatamente después del pico y una prolongada curva de agotamiento.

\subsection{Factores en la generación de crecidas}

Tras clasificar todas las crecidas en grupos homogéneos y conocer sus características, se efectuó un análisis de componentes principales. El análisis debía determinar qué grupos de variables explicaban el porcentaje más alto de la varianza. En este caso, el factor que nos interesa explicar es la respuesta hidrológica de la cuenca, medido a través del caudal. El resultado muestra que tres grupos de variables explican un total del $70 \%$ de la varianza.

Por orden de importancia, el primer componente explica un 31\%. Las variables que se hallan detrás y que están explicando la respuesta hidrológica de la cuenca son el caudal de base al inicio de la crecida y los antecedentes de precipitación más próximos en tiempo a la crecida, 1 y 3 días anteriores, es decir, el estado hidrológico de la cuenca.

El segundo componente principal explica el 21,3\% de la varianza. Este grupo de variables lo forman la precipitación registrada entre los 7 y 21 días anteriores, que aunque no es la más determinante en la generación de crecidas contribuye de forma importante a las mismas al mantener las reservas hídricas de la cuenca y favorece que la respuesta se produzca con mayor rapidez. 
El último componente explica el 16,8\% de la varianza total. En este grupo se encuentran las variables referentes a la precipitación, volumen de precipitación e intensidad (media y máxima en 5 minutos).

\section{Discusión y conclusiones}

Los resultados obtenidos informan sobre el funcionamiento hidrológico de una cuenca forestal en la montaña media pirenaica y, en general, de las áreas de montaña poco alteradas por las actividades humanas.

Es creciente la importancia que se atribuye a la vegetación en la caracterización hidrológica de las cuencas. El ya clásico estudio de Bosch \& Hewlett (1982) demostró que la introducción de cambios en la cubierta vegetal aumenta o disminuye la interceptación o el consumo de agua y, por lo tanto, determina la variabilidad espacio-temporal de la producción de escorrentía. Diversos estudios tienden, directa o indirectamente, a demostrar este paradigma a diferentes escalas (i.e. García Ruiz et al., 1995; Sahin \& Hall, 1996; Gallart y Llorens, 2002; Beguería et al., 2003; Cosandey et al., 2004; Andréassian, 2004). La mayoría de estos estudios insiste en el hecho de que un aumento de la cubierta forestal reduce el volumen de escorrentía, aunque es evidente que pueden darse excepciones relacionadas con los vientos dominantes, la exposición y las características de la vegetación.

Este trabajo no se ha centrado en la elaboración de balances hídricos que pudieran detectar la influencia de la vegetación en la generación de escorrentía. En cambio, ha podido identificar algunos rasgos hidrológicos que están directamente ligados a la presencia de una densa masa forestal. Tales rasgos son, en primer lugar, una respuesta hidrológica más retardada y, en general, menos intensa, y, en segundo lugar, un menor peso de las precipitaciones en el tipo de respuesta hidrológica, frente a una mayor responsabilidad de las condiciones antecedentes de humedad.

En un trabajo anterior se pudo comprobar que la cuenca de San Salvador tiende a reaccionar de forma muy moderada frente a las precipitaciones (García Ruiz et al., 1995). Así, en comparación con la cuenca de Arnás (localizada muy próxima, pero intensamente explotada en el pasado y casi totalmente deforestada), la cuenca de San Salvador produce siempre un pico de caudal menor, cualquiera que sea el volumen de precipitación, y su tiempo de respuesta es más lento. Además, si Arnás reacciona de forma inmediata frente a cualquier lluvia, en el caso de San Salvador se requiere una precipitación superior a 15 o $20 \mathrm{~mm}$ en 24 horas para que se produzca un aumento nítido de caudal. Sólo a finales de las estaciones húmedas y más después de una serie de sucesivas lluvias, una nueva tormenta puede dar lugar a una respuesta súbita y de gran intensidad en San Salvador (García Ruiz et al., 1995). Esta respuesta está relacionada con la existencia de suelos profundos, con los horizontes bien conservados y con elevada tasa de infiltración, que favorecen una contribución importante de escorrentía subterránea en condiciones húmedas. Además, la densa cubierta forestal intercepta una parte de la lluvia, lo que retrasa aún más el inicio de la respuesta hidrológica. 
Entre los resultados obtenidos en este trabajo, el más significativo es el limitado papel de las características de la precipitación, concretamente de la intensidad, a diferencia de lo que ocurre en otras cuencas con uso del suelo no forestal (Arnás) (García Ruiz et al., 2005). En la cuenca de San Salvador lo que realmente explica la mayor o menor intensidad de la respuesta hidrológica es el estado de humedad antecedente de la cuenca. Las lluvias anteriores (en 3, 7, 15 o 21 días) a una crecida, así como, sobre todo, el flujo de base anterior a la crecida son buenos indicadores de la intensidad de la misma. Esto sugiere que por muy intensa que sea la lluvia, en la mayor parte de los casos la cuenca tiene capacidad suficiente para absorberla, y sólo en el caso de que haya llovido en días precedentes y la cuenca esté húmeda, se producirá una respuesta de cierta intensidad. En otras palabras, la respuesta hidrológica estará más en función del estado de humedad de la cuenca que de las propias características de la precipitación. La importancia de la humedad antecedente se ha detectado también en otras cuencas, pero su peso no llega a ser tan determinante, como se ha comprobado en la cuenca de Arnás (García Ruiz et al., 2005) y en las cuencas experimentales del Alto Llobregat (Gallart et al., 1998).

\section{Agradecimientos}

Este trabajo ha sido realizado gracias a los siguientes proyectos: "Hydrological processes in Pyrenean catchments in relation to land-use changes and climatic fluctuations" (PIRIHEROS, REN 2003-08678/HID) y "Characterization and modelling of hydrological processes and regimes in gauged basins for the prediction in non-gauged basins" (CANOA, CGL 2004-04919-C02-01). La monitorización de la cuenca ha sido posible gracias al consorcio entre CSIC y el Ministerio de Medio Ambiente (Proyecto RESEL). La contribución del primer autor ha sido posible gracias a una beca predoctoral con cargo al Proyecto: Procesos hidrológicos y erosivos en cuencas pirenaicas en relación con los cambios de usos del suelo y la variabilidad climática. Por último los autores desean expresar su agradecimiento a Sergio Valdivielso, Eugenio Mingo y Ethel Navarro en el trabajo de campo.

\section{Referencias bibliográficas}

ANDRÉASSIAN, V. (2004). Water and forest: from historical controversy to scientific debate. Journal of Hydrology, 291: 1-27.

Beguería Portugues, S., Lopez-Moreno, Ji., Lorente, A., Seeger, M. y García-Ruiz, JM. (2003). Assesing the Effect of Climate Oscillations and land-Use Changes on Streamflow in the Central Spanish Pyrenees. Ambio 32: 283-286.

Beven, K. (1991). Hydrograph separation? Proceedings of the 3rd National Hydrology Symposium, Southampton, U.K. 3.1-3.7. 
Bosch, J. y Hewlett, JD. (1982). A review of catchment experiments to determine the effect of vegetation changes on water yield and evapotranspiration. Journal of Hydrology, 55: 3-23.

Brown, A., Zhang, L., McMahon, TA., Western, AW. y Vertessy, RA. (2005). A review of paired catchment studies for determining changes in water yield resulting from alterations in vegetation. Journal of Hydrology, 310: 28-61.

Cosandey, C., Andréassian, V., Martin, C., Didon-Lescot, JF., Lavabre, J., Folton, N., Mathys, N. y Richard, D. (2004). The hydrological impact of the mediterranean forest: a review of French research. Journal of Hydrology, 301: 1-15.

Creus, J. y GiL, M. (2001). Clima. En: El medio físico y su peligrosidad en un sector del Pirineo Central. Madrid, Instituto Geológico y Minero de España. Ministerio de Ciencia y Tecnología.

Del Barrio, G., Creus, J. y Puigdefabregas, J. (1990). Thermal seasonality of the high mountain belts of the Pyrenees. Mountain Research and Development, 10: 227-233.

Gallart, F., Llorens, P., Latron, J., Regüés, D. y Salvany, C. (1998). Las cuencas experimentales de Vallcebre (I): funcionamiento hidrológico. En: Investigaciones recientes de la Geomorfología española (A. Gómez Ortiz, Salvador Franch, F. eds.). Universitat de Barcelona, Sociedad española de Geomorfología, Universidad de Granada. pp: 187-192. Granada.

Gallart, F. y Llorens, P. (2002). Water resources and environmental change in Spain. A key issue for sustainable integrated catchment management. In: Environmental change and wayer sustainability (J.M. García Ruiz, J.A.A. Jones \& J. Arnáez, eds.). Instituto Pirenaico de Ecología, pp.11-20. Zaragoza.

García Ruiz, J. y Puigdefábregas-Tomás, J. (1982). Formas de erosión en el Flysch Eoceno surpirenaico. Cuadernos de Investigación Geográfica. 8: 83-126.

García Ruiz, J., Puigdefábregas-Tomás, J. y Creus, J. (1985). Los recursos hídricos superficiales del Alto Aragón. Instituto de Estudios Altoaragoneses. 224 p. Huesca.

García Ruiz, J.M., Lasanta, T., Ortigosa, L., Ruiz Flaño, P., Martí, C. y González, C. (1995): Sediment yield under different land-uses in the Spanish Pyrenees. Mountain Research and Development, 15 (3): 229-240.

GARCíA-Ruiz, J. M. y GALlART, F. (1997). Las cuencas experimentales como base para el estudio de la erosión y la desertificación. En: El paisaje mediterráneo a través del espacio y del tiempo. Implicaciones en la desertificación (J. Ibañez, Valero Garcés, B., Machado, C. eds.). Geoforma Ediciones, pp. 221-238, Logroño.

García-Ruiz, J., Arnaez, J., Beguería, S., Seeger, M., Martí-Bono, C., Regüés, D., Lana-Renault, N. y White, S. (2005). Runoff generation in a intensively disturbed, abandoned farmland catchment, Central Spanish Pyrenees. Catena, 59: 79-92. 
Hewlett, J.D. (1982). Principles of forest hydrology. University of Georgia Press, 183 pp.

HibBert, A. (1967). Forest treatment effects on water yield. In: International Symposium for Hydrology (W.E. Sopper \& H.W. Lull eds.). Pergamon, Oxford, 813 pp.

Monserrat Recoder, P. (1971). El ambiente vegetal jacetano. Pirineos, 101: 5-22.

Seeger, M., Errea, MP., Beguería S., Arnáez, J., Martí-Bono, C. y García-Ruiz, JM. (2004). Catchment soil moisture and rainfall characteristics as determinant factors for dischargue/suspended sediment hysteretic loops in a small headwater catchment in the Spanish Pyrenees. Journal of Hydrology, 288: 299-311.

SAHIN, V. y Hall, M.J. (1996). The effects of afforestation and deforestation on water yields. Journal of Hydrology, 178 (1-4): 293-309. 